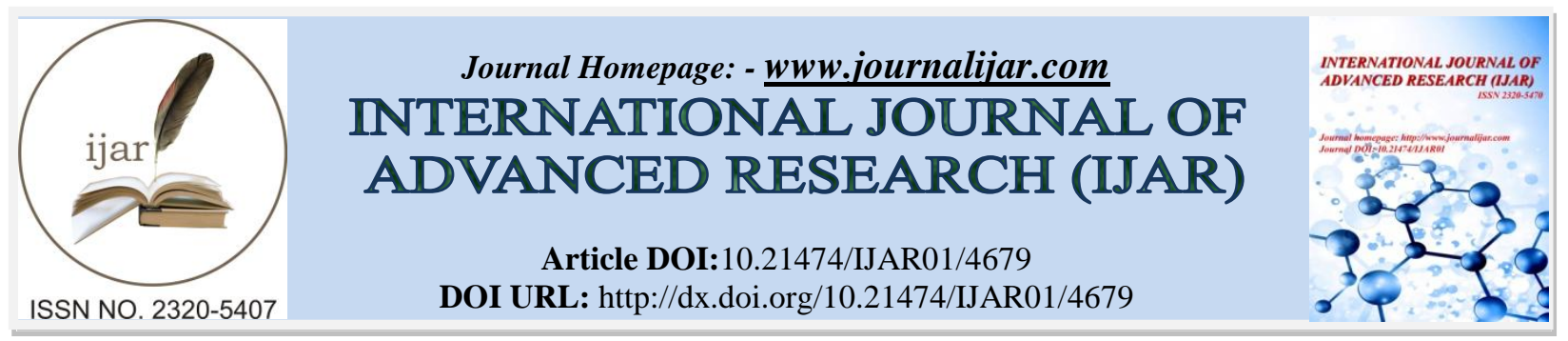

RESEARCH ARTICLE

\title{
NEUTROPHIL EXPRESSION OF CD64 IN EARLY DIAGNOSIS OF EARLY ONSET NEONATAL SEPSIS.
}

\author{
Deena A. Elshabrawy ${ }^{1}$ and Yehia H. Abdel-maqsoud ${ }^{2}$ and Elzahraa Arafat ${ }^{3}$. \\ 1. Deena Elshabrawy, Assistant professor of Clinical Pathology, University of Benha, Faculty of Medicine, Egypt. \\ 2. Assistant Prof. of Pediatric and neonatology Benha University, Faculty of Medicine, Egypt. \\ 3. M.B.B.Ch., Msc.
}

\section{Manuscript Info}

Manuscript History

Received: 25 April 2017

Final Accepted: 27 May 2017

Published: June 2017

Key words:-

CD64, Neutrophils, Early Onset

Neonatal Sepsis

\section{Abstract}

The diagnosis of sepsis remains one of the most difficult tasks for physicians and other medical staff. Blood cultures often remain negative in the presence of pneumonia, meningitis and even fulminant blood born septicemia. A rapid laboratory test with high specificity for neonatal sepsis would be a valuable tool in therapeutic decision making and avoiding the unnecessary use of antibiotics in patients with clinical signs and symptoms of sepsis but negative blood cultures. The rapid upregulation of CD64 expression on the polymorphnuclear neutrophils (PMNs) and the normal 6-hour half-life of the blood PMNs made the determination of the PMN CD64 expression a true indication of the current status of neonatal sepsis. In this study, we selected the CD64 to detect its value in the diagnosis of early-onset neonatal sepsis cases by using the flow cytometry. We aimed to evaluate its association with the different demographic, clinical and laboratory data. The current study was carried out on 100 patients; 40 diagnosed early onset neonatal sepsis (EONS) (culture + ve) neonates, 40 clinically suspected EONS (culture -ve) and 20 neonates (healthy or admitted for jaundice) as a control group. All patients were subjected to complete history taking, thorough clinical examination and laboratory investigations. We found that; CD 64 expression was only positive in both EONS groups but not in control group. CD64 expression was significantly higher in EONS (culture + ve) group than in suspected EONS (culture -ve). It has high sensitivity $(80 \%)$ and intermediate +ve predictive value (53\%), but low specificity (10\%). We found that CD64 expression had no correlation to any of the clinical or laboratory findings except for the temperature and CRP. when we used the ROC curve to compare the area under the curve between the CD64 \& CRP we found that the area under the curve (accuracy) for CD64 $=0.855$, while area under the curve (accuracy) for CRP $=0.493$. This implies the greater diagnostic power for CD64 than CRP for early detection of EONS.

Copy Right, IJAR, 2017,. All rights reserved. 


\section{Introduction:-}

Sepsis in neonates hospitalized in the neonatal intensive care unit (NICU) is a global problem and is a significant contributor to morbidity and mortality. Most studies focus on infants of very low birth weight (VLBW), given their immature immune system and the added contribution of a variety of risk factors [1]. It is important to realize that even late-preterm infants have a compromised immune system and are susceptible to infections in the NICU [2].

Isolation of bacteria from a central body fluid (usually blood) is the standard and most-specific method to diagnose neonatal sepsis; however, positivity rates vary widely, ranging from 30 to $87 \%$. Physicians must therefore rely on a variety of nonspecific laboratory tests to assist in and perhaps speed diagnosis of sepsis. The microorganisms most commonly associated with early-onset infection include Escherichia coli, Klebsiella, coagulase-negative Staphylococcus, Haemophilus influenza, Group B Streptococci (GBS), Listeria monocytogenes, Candida and mixed infections [3].

C-reactive protein (CRP) is probably the single most commonly used laboratory test for early diagnosis of neonatal sepsis. It is however, well known that the CRP concentration does not rise very early during an infection and a low value does not rule out sepsis. The production of CRP does not start until 6 - 8 hours after the onset of the infection [4]

Two or more of the following clinical criteria were used to identify patients for sepsis evaluations: respiratory compromise, that is, tachypnea, increased apnea or severe apnea requiring positive pressure ventilation; cardiovascular compromise, that is, bradycardia, pallor, decreased perfusion or hypotension; metabolic changes, that is, hypothermia, hyperthermia, feeding intolerance glucose instability, or metabolic acidosis ; or neurologic changes, that is, lethargy, hypotonia, or decreased activity. These clinical features were validated to be strongly suggestive of infection [5].

Two or more of the following previously validated hematologic criteria were used as indicators for bacterial sepsis: total leucocytic count of $<5000$ or $>25000$ cells per mm3, absolute neutrophil count (ANC) of $<1500$ cells per mm3, immature/total neutrophil (I/T) ratio of $>0.16$, platelet count of $<150000$ cells per mm3 and CRP $>10 \mathrm{mg} / \mathrm{l}$ [6]. Infants with negative culture results and with a positive sepsis score $(<2$ of the clinical criteria and $<2$ of the hematologic criteria enumerated above) were categorized as having clinical sepsis. Proven neonatal sepsis included cases where in addition to clinical and laboratory signs, a pathogen was isolated from a normally sterile body fluid [5].

One of the effects of inflammatory cytokines on the innate immune response is the rapid up-regulation of CD64 expression on the neutrophil membrane. When neutrophils detect an infection, they become activated, and produce a protein called CD64 (a cell marker) on their surface. Neutrophils produce the CD64 within 1 hour of detecting an infection [7].

CD64 measures the up regulation of an Fc receptor (Fc gamma R1, or CD64) on neutrophils. The Fc receptors on white blood cells are very important for effective phagocytosis of bacteria and are upregulated during infections [5]. The high affinity of CD64 is mainly involved in phagocytosis and intracellular killing of pathogen, but it is also expressed at very low levels on the surface of unstimulated neutrophil [8]. In addition, it has been shown that neutrophils of pre-term infants express CD64 antigen to a similar extent comparing to older children and adults. Thus, this specific marker can be used for the identification of life threatening infections in preterm infants [9].

In this study, we selected the CD64 to detect its value in the diagnosis of early-onset neonatal sepsis cases by using the flow cytometry. We aimed to evaluate its association with the different demographic, clinical and laboratory data.

\section{Patients and Methods:-}

This study was carried on 80 patients with early onset neonatal sepsis, attending the BenhaTeatching Hospital. They were 36 males $(45 \%)$ and 44 females (55\%). 40 of them (28 males and 12 females) diagnosed clinically and laboratory as EONS and the other 40 neonates (20 males and 20 females) suspected clinically as EONS but, their blood cultures were negative (their ages ranged from 0 day to 7 days) and 20 controls (healthy or admitted for jaundice) with matched age and sex. 


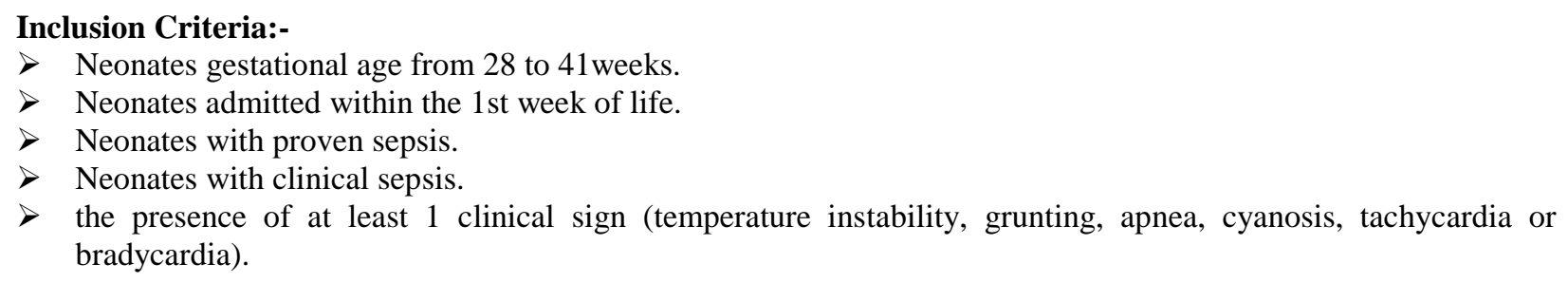

\section{Exclusion Criteria:-}

$>$ Neonates with multiple congenital anomalies.

$>$ Neonates with genetic syndromes.

$>$ Neonates admitted after the 1 st week of life.

$>$ Infants with neonatal asphyxia (Apgar score $<7$ at 5 minutes).

All patients are subjected to clinical history, thorough clinical examination and laboratory investigations, including complete blood count (CBC), blood culture, c-reactive protein (CRP). For assessment of neutrophil CD64, whole blood samples were analyzed within 24 hours. Leukocytes $(1 \times 106)$ were labeled with the appropriate antibody for 15 minutes at room temperature in darkness. Erythrocyte lysis was performed using Pharm Lyse (Becton Dickinson), a buffered ammonium chloride-based lysing solution, according to the manufacturer's recommendations, then washed in BD Cell Wash (BD Biosciences). A minimum of 10000 neutrophil-gated events was acquired for each patient Positivity was defined as $>20 \%$ of neutrophil cells expressing a given surface antigen (CD64). A sequential gating strategy for the neutrophils using multicolor flow cytometry was established using Coulter EPICS-XL, USA. Informed consents were obtained from parents of patients who served as subjects in this study.

\section{Results:-}

The current study showed that the most common organisms in septicemic group were gram negative organisms (30\%) then gram positive organisms (17.5\%) then fungal infection (2.5\%) (table1).

CD 64 was expressed in all EONS 40(40\%) (culture +ve) group, no negative cases was detected. While in the clinically suspected EONS (culture -ve) group, CD 64 was only expressed in $28(28 \%)$ patients. No neonates in the control group showed positive CD 64 expression (table 2). CD 64 expression was highly significant higher in both, EONS group and clinically suspected group than in the control group $(\mathrm{p}<0.001)$. CD64 expression was also higher in the EONS (culture +ve) group than in the suspected EONS (culture -ve) group ( $\mathrm{p}<0.05$ ) (table 3 ). No statistically significant associations were elicited between CD64 expression and both EONS groups concerning the age, sex, gestational age and birth weight ( $p>0.05)$. There was statistically positive significant association elicited between CD64 expression and the temperature of the patients $(p<0.05)$ while, no significant correlation was found between CD64 expression and other clinical data as Respiratory rate (RR) \& heart rate (HR) (table 4). No significant associations were found between CD64 expression in the EONS groups regarding the TLC, HB, PLT, Staff, Segment neutrophils and immature to total ratio of the neutrophils (I/T ratio) $(\mathrm{p}>0$. 05). Our results showed that, there was statistically significant Correlation between CD64 expression and the CRP in both EONS groups ( $\mathrm{p}=0$. 05) (Table 5). The ROC curve (Table 6) (Figure 1), showed that, although the CD 64 expression has low specificity $(10 \%)$ but, it has high sensitivity $(80 \%)$ and intermediate +ve predictive value $(53 \%)$, This high sensitivity indicates the usefulness of CD64 in differentiation between neonatal sepsis and non-infected cases. Furthermore, when we used the ROC curve to compare the area under the curve between the CD64 \& CRP we found that the area under the curve (accuracy) for CD64 $=0.855$, while area under the curve (accuracy) for CRP $=0.493$. This implies the greater diagnostic power for CD64 than CRP for early detection of EONS (figure2). 
Table 1:- Causative organisms among septicemia groups:

\begin{tabular}{|c|c|c|}
\hline Blood culture & NO. & \% \\
\hline Gram positive organisms & $\mathbf{1 4}$ & $17.5 \%$ \\
\hline CONS & 4 & $5 \%$ \\
Staph. Aureus & 4 & $5 \%$ \\
GBS & 4 & $5 \%$ \\
Strept. pneumonia & 2 & $2.5 \%$ \\
\hline Gram negative organisms & $\mathbf{2 4}$ & $30 \%$ \\
\hline E. coli & 8 & $10 \%$ \\
Klebsiella & 14 & $17.5 \%$ \\
Pseudomonus & 2 & $2.5 \%$ \\
\hline Fungal infection & $\mathbf{2}$ & $2.5 \%$ \\
\hline Candida albican & 2 & $2.5 \%$ \\
\hline No growth & $\mathbf{4 0}$ & $50 \%$ \\
\hline Total & $\mathbf{8 0}$ & $100 \%$ \\
\hline
\end{tabular}

Table 2:- CD64 expression in the studied groups.

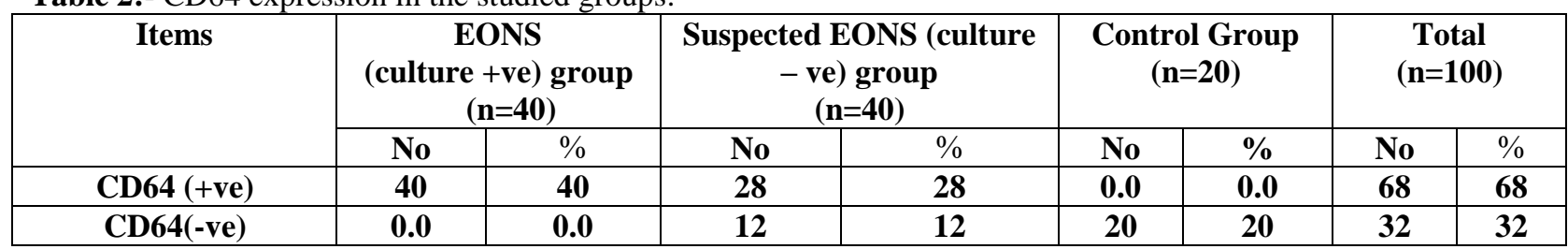

Table 3:-Comparison between CD64 expression in different studied groups.

\begin{tabular}{|c|c|c|c|c|c|c|c|c|}
\hline & Group & Mean & SD & MIN & MAX & f- test & $\begin{array}{c}\text { p } \\
\text { value }\end{array}$ & Significance \\
\hline \multirow{3}{*}{$\begin{array}{c}\text { CD64 } \\
(\%)\end{array}$} & EONS & $\mathbf{6 2 . 1 5}$ & $\mathbf{2 8 . 6 2}$ & $\mathbf{2 3 . 5}$ & 99 & \multirow{2}{*}{27.66} & $\mathbf{0 . 0 0 1}$ & \multirow{2}{*}{ HS } \\
\cline { 2 - 7 } & $\begin{array}{c}\text { Suspected } \\
\text { EONS }\end{array}$ & $\mathbf{4 8 . 2 6}$ & $\mathbf{1 6 . 2 5}$ & $\mathbf{2 6 . 9}$ & $\mathbf{8 0}$ & & & \\
\cline { 2 - 6 } & $\begin{array}{c}\text { Control } \\
\text { Group }\end{array}$ & 2.39 & 1.17 & 1.1 & 5.1 & & & \\
\hline
\end{tabular}

EONS group and control group $=\mathrm{HS}$

Suspected EONS group and control group = HS

Table 4:-Correlation between the CD64 and some Clinical data in EONS groups:

\begin{tabular}{|c|c|c|c|c|}
\hline \multirow{3}{*}{ CD64 (\%) } & parameters & Rcorrelation & p value & Significance \\
\cline { 2 - 5 } & RR(/min) & $\mathbf{0 . 0 4 5}$ & $\mathbf{0 . 8 6}$ & NS \\
\cline { 2 - 5 } & HR (beat/min) & $\mathbf{0 . 2 1 2}$ & $\mathbf{0 . 3 7}$ & NS \\
\cline { 2 - 5 } & Temperature $\left({ }^{\mathbf{c}} \mathbf{c}\right)$ & $\mathbf{0 . 5 1}$ & $\mathbf{0 . 0 2}$ & $\mathrm{S}$ \\
\hline
\end{tabular}

Table 5:- Correlation between the CD64 and CRP in EONS groups.

\begin{tabular}{|c|c|c|c|c|}
\hline \multirow{2}{*}{$\begin{array}{c}\text { CD64 }(\%) \\
\text { EONS } \\
\text { (culture +ve) group }\end{array}$} & parameters & r correlation & P value & Significance \\
\cline { 2 - 5 } & CRP(mg/l) & 0.45 & 0.05 & S \\
\hline $\begin{array}{c}\text { CD64 }(\%) \\
\text { culture -ve) group }\end{array}$ & CRP(mg/l) & $\mathbf{0 . 4 5}$ & $\mathbf{0 . 0 5}$ & $\mathrm{S}$ \\
\hline
\end{tabular}

Table 6:-Sensitivity\& specificity of neutrophil CD64 expression using ROC curve.

\begin{tabular}{|c|c|c|c|c|c|}
\hline $\begin{array}{c}\text { Area under the curve } \\
\text { (accuracy) }\end{array}$ & Cut off point & sensitivity & specificity & $\begin{array}{c}\text { Predictive } \\
\text { value +ve }\end{array}$ & $\begin{array}{c}\text { Predictive } \\
\text { value -ve }\end{array}$ \\
\hline $\mathbf{0 . 6 1 8}$ & $\mathbf{2 9 . 2}$ & $\mathbf{8 0 \%}$ & $\mathbf{1 0 \%}$ & $\mathbf{5 3 \%}$ & $\mathbf{3 3 \%}$ \\
\hline
\end{tabular}




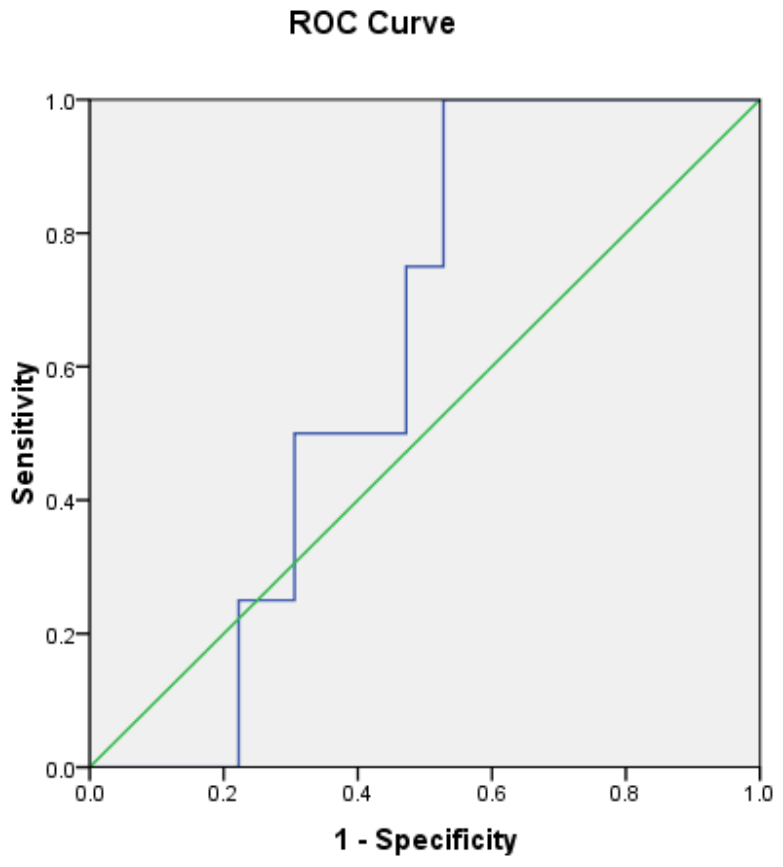

Figure 1:- Sensitivity\& specificity of neutrophil CD64 expression using ROC curve.

\section{Discussion:-}

The diagnosis of sepsis remains one of the most difficult tasks for physicians and other medical staff. Blood cultures often remain negative in the presence of pneumonia, meningitis and even fulminant blood born septicemia. Capturing the specific organism in a small sample of venous blood remains elusive. A rapid laboratory test with high specificity for neonatal sepsis would be a valuable tool in therapeutic decision making and avoiding the unnecessary use of antibiotics in patients with clinical signs and symptoms of sepsis but negative blood cultures [10] In this study, we tried to determine the CD64 expression as an immunological marker for rapid diagnosis of neonatal sepsis. We tried to elucidate the value of CD64 expression on neutrophil surface in EONS using the flow cytometry. The significance of CD64 expression was investigated in relation to various clinical, laboratory and standard prognostic factor (CRP).

In this study, there were high statistical significant differences in mean CD64 expression levels among studied groups $(\mathrm{p}<0.005)$ (Table 3). The percentage of +ve CD64 expression in the studied groups was $(68 \%)$, while the percentage of -ve CD64 expression was (32\%) (Table 2). These findings were in agreement with previous study, showed raised percentages of CD64+ cells in proven and clinical sepsis group [11]. Also, similar results have been reported in adults [8\&12] and neonates [13]. These findings were confirmed by other study which revealed that patients with sepsis had a greater number of circulating CD64 positive PMNs (mean 71\%) than in healthy controls (mean 19\%), This mean that an increase in the number of CD64 molecules expressed on the PMN surface compared to healthy individuals [14].

In the current study, positive CD64 expression showed no significant association with other clinical features as respiratory rate and heart rate but there was significant association between CD64 and temperature of the patient. This was in a concordance with other study which reported that, increase CD64 expression was not correlated with any clinical variables except the temperature [11]. Also, our results confirmed by previous studies performed in adults [14\&15] and in neonates [16\&17].

There were no significant correlations of the hematological data (TLC, staff, segment, HB, platelets and immature to total ratio) of the patients and CD64 in the present study patients. These results are in agreement with other previously reported results [11\&17]. Thrombocytopenia is one of the most common complications of neonatal sepsis; this may be attributed to bone marrow depression, consumption coagulopathy, platelet sequestration or combination of these processes [18]. The finding in the present study showed no significant correlation between 
both CD64 and platelet count which does not exclude CD64 as a parameter of the severity of sepsis. This may be due to the small sample size. This was in agreement with previous study [19].

In the current study, CD64 expression had a significant correlation with CRP level which is a laboratory marker of neonatal sepsis pointing to its usefulness as additional marker of sepsis. other studies correlated Neutrophil's CD 64 positively with CRP [20\&21]. In this work, ROC curve shows area under the curve (accuracy) for CD64 greater than area under the curve (accuracy) for CRP. This implies the greater discriminating power for CD64 than CRP for early detection of EONS. This was in agreement with previous studies [22\&23], yet another study, on 92 EONS patients, found that CD64 expression had the greater discriminating power than CRP [24].

In our study, we found $80 \%$ sensitivity and $10 \%$ specificity for CD64 expression, on the other hand, very high sensitivity and specificity for CD64 had been reported in early onset sepsis as 97\% and 89\%, respectively [19],. Another study, showed that sensitivity and specificity of CD64 in early onset sepsis is $97 \%$ and $72 \%$, respectively [12\&13]. In addition, we obtained the positive and negative predictive value (PPV and NPV) of CD64 as 53\% and $33 \%$, respectively. This was in contrast to other works which showed high PPV and NPP for CD64 in early onset sepsis, about $87 \%$ and $72 \%$, respectively [13\&22]. The discrepancy between the results may be attributed to small sample size of our cases.

In this study, we found that the enhanced expression of CD64 by neutrophils has a high sensitivity and intermediate positive predictive value for neonatal sepsis, but a low specificity. It is worth mentioning that the analysis of CD64 expression is performed with a small sample $(25-50 \mu \mathrm{l}$ of whole blood), is very rapid $(<20 \mathrm{~min})$, and highly reliable. In addition, our data indicate that the high sensitivity of raised levels of CD64 for neonatal sepsis is achieved through a single determination and the high percentage of CD64+ cells seen in EONS is maintained in these patients for at least 6 hours [10]. We concluded that, measurement of neutrophil surface marker CD64 can be useful for diagnosis of infection in early phase.

Disclosure of conflicts of interest:-We declare that we have no conflicts of interest to disclose.

Ethical approval:- "All procedures performed in studies involving human participants were in accordance with the ethical standards of the Benha university Faculty of medicine research committee and with the 1964 Helsinki declaration and its later amendments."

Informed Consent:- "Informed consent was obtained from all individual participants included in the study."

\section{Referrences:-}

1. Bizzarro MJ, Raskind C, Baltimore RS and Gallagher PG: "Seventy-five years of neonatal sepsis at Yale: 19282003". Pediatrics;(2005) 116:595-602.

2. Benjamin DK: "Infection in late preterm infants". J ClinPerinatol; (2006), 33:871-882.

3. Caldas JP and Oliveira RT: "Accuracy of white blood cell count and C-reactive protein, for diagnosing early neonatal sepsis". J Pediatr (Rio J); (2008), 84:536-42.

4. Sabel KG and Wadsworth C): "C-reactive protein (CRP) in early diagnosis of neonatal septicemia". J ActaPaediatrScand; (1999), 88:825-31.

5. Bhandari V, Wang C, Rinder $\mathrm{C}$ and Henry H: "Hematologic profile of sepsis in neonates:Neutrophil CD64 as a diagnostic marker". Pediatrics; (2008), 121(1): 129-34.

6. Karen M: "Bacterial and fungal infections". In: Cloherty P, Eric C, Stark R, eds. Manual of Neonatal Care, 6th edition. (2008), Lippincott Williams \&Wilkins; 279.

7. Davis B, Karen C and Rinder H: "CD64 expression in early onset sepsis". J Critcal Care; (2008) S4:20-26.

8. Fjaertoft G, Pauksen K, Hankansson L, Xu S and Venge P: "Cell surface expression of CD64 on neutrophils and monocytes in patients with influenza A with or without complications". Scand J Infect Dis; (2010), 37 (1112): 882-9.

9. Rosales WC, Akisu M, Cetingul N and Caglayan S: "Reactive hyperemia and interleukin 6, interleukin 8, and tumor necrosis factor- $\alpha$ in the diagnosis of earlyonset neonatal sepsis. Pediatrics"; (2011) 108 (4): E61.

10. Baranda L, Layseca-Espinosa E, Perez-Gonzalez LF, Rosenstein Y et al.: Expression of CD64 as a potential marker of neonatal sepsis. Pediatr Allergy Immunol.; (2010), 13:319-27. 
11. Espinosa E, Perez-Gonzalez LF, Torres-Montes A, Baranda L, de la Fuente H, Rosenstein Y et al. (: Expression of CD64 as a potential marker of neonatal sepsis. Pediatr Allergy Immunol; (2009) 13:319-27.

12. Lino S, Ravetch R, Kint JP, Perussia F and Bazil V: Cell adhesion molecules in inflammatory diseases. Drugs; (2009) 56: 977-88.

13. Herra G, Groselj-Grenc M, Ihan A and Derganc M: Neutrophil and monocyte CD64 and CD163 expression in critically ill neonates and children with sepsis: comparison of fluorescence intensities and calculated indexes. Mediators Inflamm; (2008), 2026-46.

14. Qureshi S, Lewis M, Gant V, et al., Increased distribution and expression of CD64 on blood polymorphonuclear cells from patients with the systemic inflammatory response syndrome (SIRS). Clinical and Experimental Immunology. (2011), 125(2):258-265.

15. Allen S, Karstaedt M, Manikaut K and Heather H: "Pneumococcal bacteremia during a decade in children in Soweto, South Africa". Pediatr Infect Dis J; (2009) 19:454-457.

16. Ng PC, Li G, Chui KM, Chu WC, Li K and Wong RP: "Neutrophil CD64 is a sensitive diagnostic marker for early-onset neonatal infection". Pediatr Res; (2009), 56 (5): 796-803.

17. Livaditi O, Kotanidou A, Psarra A, Dimopoulou I, Sotiropoulou C, Augustatou K, et al., Neutrophil CD64 expression and serum IL-8: sensitive early markers of severity and outcome in sepsis. Cytokine; (2010), 36 (56): 283-90.

18. Wong W and Glader B: Approach to the Newborn Who Has Thrombocytopenia. Neoreviews; (2010), 5 (10): p444-50.

19. Gonzalez BE, Mercado CK, Johnson L, Brodsky NL Bhandari V: Early markers of late onset sepsis in premature neonates: clinical, hematological and cytokine profile. J Perinat Med; (2009), 31: 60 -8.

20. Kantar M, Kultursay N, Kutukculer N, Akisu M, Cetingul N Caglayan S: Plasma concentrations of granulocytemacrophage colonystimulating factor and interleukin-6 in septic and healthy preterms. Eur J Pediatr; (2009), 159: $156-7$.

21. Martin H, Olander B and Norman M) : Reactive hyperemia and interleukin 6, interleukin 8, and tumor necrosis factor- $\alpha$ in the diagnosis of earlyonset neonatal sepsis. Pediatrics; (2010),108 (4): E61.

22. Lichtman MA and Liesveld JL: Comparison of procalcitonin with CD64, C-reactive protein and differential white blood cell count for the early diagnosis of bacterial infections in newborn infants. Pediatr Infect Dis J; (2009), 18: 666-671.

23. Lee M, Tan T and Feng A: "Clinicopathologic analysis of early onset neonatal sepsis in a single institution". Journal of the Chinese Medical Association; (2010), 70(7): 269.

24. Vercauteren SM and Sutherland HJ: Predictive value of soluble immunological mediators in neonatal infection. ClinSci (Lond); (2011), 87:165-171. 